\title{
Use of FIB to Identify Opens in Metal - Metal Bonds
}

\author{
Nathan Wang, Jason Silva, and George Perreault \\ Maxim Integrated, San Jose CA, USA
}

Metal to metal bonds are used to make connections to integrated circuits through die attach, wire bonding, or chip scale packaging techniques. Regardless of the materials and how the connections are formed, they all have one thing in common; they sometimes fail open.

Although the FIB (Focused Ion Beam) is the cross sectioning tool of choice in failure analysis [1], it is not often used in these applications. A typical FIB cross section is $20 \mu \mathrm{m}$ wide and 5-8 $\mu \mathrm{m}$ deep but a ball bond is $75-100 \mu \mathrm{m}$ tall and $\sim 30 \mu \mathrm{m}$ thick and solder balls are typically at least $400 \mu \mathrm{m}$ in diameter. Even with the latest high throughput machines, FIB cross sectioning of these open failures is simply not feasible. Although IC package cross sectioning can't realistically be done in a FIB, it is still an indispensable tool for these types of analyses.

Open IC failures are typically mechanically cross sectioned through the suspected open site. Because most metals, particularly gold, are soft and smear easily during polishing, post lapidary processing steps are typically used before inspecting the suspected failure location. Traditional metallography techniques involve liquids to lightly etch the polished surface [2]. These techniques are both easy and quick, but etch rates, and therefore effectiveness, vary greatly from sample to sample. In addition to the etch rate control issue, these liquids (typically acids) contaminate the sample making subsequent elemental analyses problematic.

An issue with broken security bonds was observed. As part of the investigation, unstressed bonds were examined to look for possible assembly issues. A metal etch (Potassium Iodide based Columbus Chemical Industries Palladium Etch) was used to decorate the interfaces, Figure 1. The chemical was applied with a cotton swab and then dropped in a beaker of DI water after the etch was completed. After only 3 seconds, damage to the nickel plating layer was observed. After six seconds, the copper lead frame and gold wire bond were also affected.

As these results were not satisfactory, they were repeated with the use of a FIB. To clean the sample, the beam current was maximized and the sample was orientated so there was a $5^{\circ}-15^{\circ}$ angle between the sample surface and the ion beam. If a dual beam FIB is used, progress can be monitored with the electron beam. Depending on the area, cleaning takes between 1-10 minutes.

Results of the FIB cleaning of the security bonds are included in Figure 2. Unwanted material was removed, including the dark polishing grit buried in the soft gold, but none of the layers were chemically attacked, allowing follow up elemental analyses to look for possible contaminants.

An open was identified on a two terminal device; one connection was wired bonded, the second was made through die attach. The device was mechanically cross sectioned, but due to metal smearing, no definitive conclusion could be reach. FIB cleaning was subsequently performed on both the wire bond and die attach interfaces. These results confirmed that the wire bond, and not the die attach, was the root cause of the failure, Figure 3.

Metal to metal bonds will always be used in the semiconductor industry and some small number of them will fail. To reach the proper conclusion and to allow subsequent chemical analyses, FIB sample cleaning, and not metal etching, is recommended to remove sample preparation artifacts. 


\section{References}

[1] S. Reyntjens, J. Micromech. Microeng. 11 (2001) p. 287.

[2] E.C. Olden, Symp. Methods of Metallographic Specimen Preparation, ASTM, (1960), p. 16.

[3] K. Ohya, "Imaging Using Electrons and Ion Beams", Focused Ion Beam Systems, ed. N. Yao, Cambridge, (2007), p. 108.
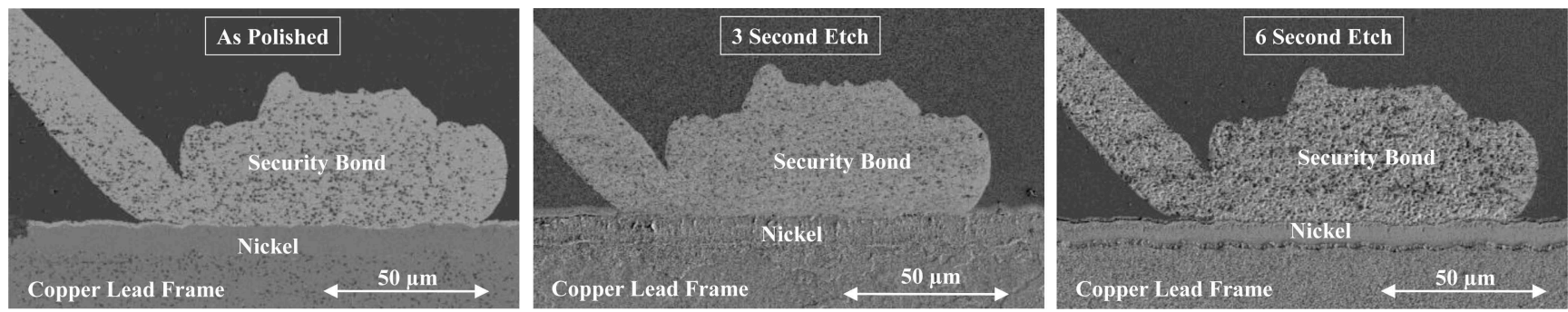

Figure 1: SEM images of a cross sectioned gold security bond. a) As polished. b) After three second chemical etch. C) After a six second chemical etch.
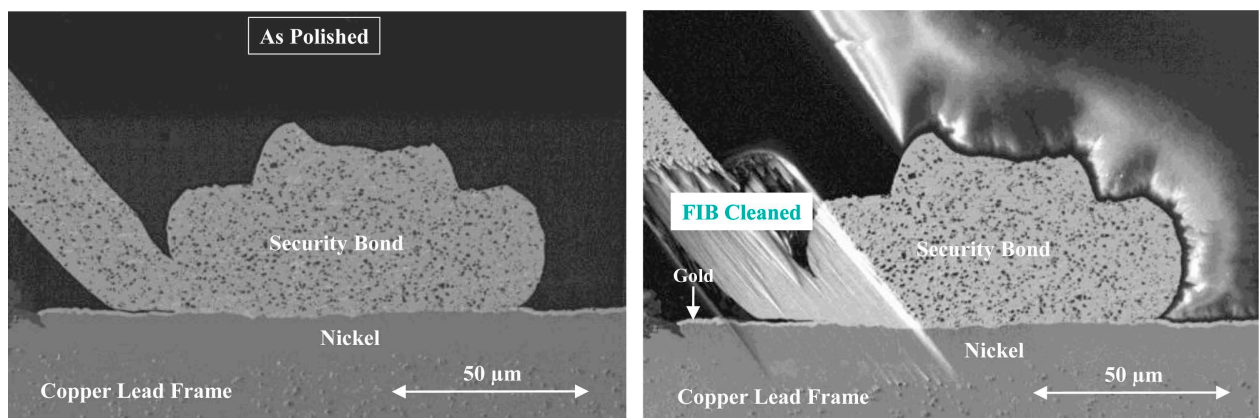

Figure 2: SEM images of a cross sectioned gold security bond. a) As polished. b) After a FIB clean. The interface of interest has been cleared of debris without chemically changing any of the layers
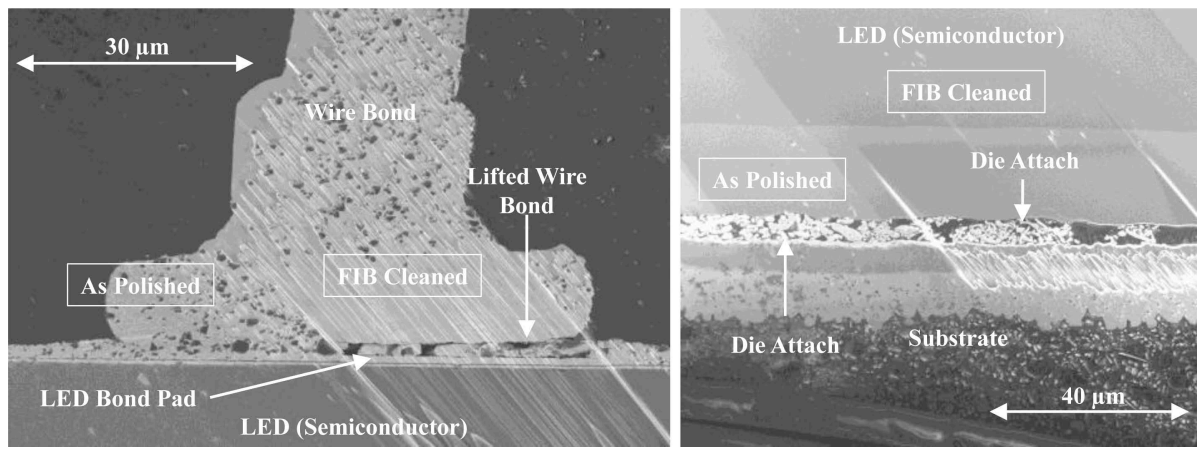

Figure 3: Opens investigation of a two terminal device. After FIB cleaning, it was clear that the bond wire, not the die attach, was the root cause of the failure. 